\title{
Non-Markovian dynamics of collective atomic states coupled to a waveguide
}

Sinha, Kanu, Meystre, Pierre, Solano, Pablo

Kanu Sinha, Pierre Meystre, Pablo Solano, "Non-Markovian dynamics of collective atomic states coupled to a waveguide," Proc. SPIE 11091, Quantum Nanophotonic Materials, Devices, and Systems 2019, 1109100 (3 September 2019); doi: 10.1117/12.2530927

EPIE Event: SPIE Nanoscience + Engineering, 2019, San Diego, California, United States 


\title{
Non-Markovian dynamics of collective atomic states coupled to a waveguide
}

\author{
Kanu Sinha ${ }^{\mathrm{a}}$, Pierre Meystre ${ }^{\mathrm{b}}$, and Pablo Solano ${ }^{\mathrm{c}}$ \\ ${ }^{a}$ US Army Research Laboratory, Joint Quantum Institute, University of Maryland, College \\ Park, USA \\ ${ }^{\mathrm{b}}$ Department of Physics and College of Optical Sciences, University of Arizona, Tucson, USA \\ ${ }^{\mathrm{c}}$ Department of Physics, MIT-Harvard Center for Ultracold Atoms, and Research Laboratory \\ of Electronics, Massachusetts Institute of Technology, Cambridge, USA
}

\begin{abstract}
When atoms are optically coupled to a one dimensional waveguide, they can interact through macroscopic distances. The retardation effects inherent to field propagation and the associated delay in information backflow between the atoms result in a departure from the familiar Markovian dynamics. We study the case of two twolevel atoms coupled along a waveguide. One remarkable feature of the dynamics in this regime is the formation of long-lived bound states in the continuum (BIC), ${ }^{30}$ that refer to a hybrid diatomic molecule bound together by propagating modes of a field. In particular, we study the probability of reaching such a bound states of the system starting in an initially anti-symmetric state of the emitters.
\end{abstract}

Keywords: Waveguide Quantum Electrodynamics (QED), Non-Markovian dynamics, Time-delayed feedback, Dicke superradiance

\section{INTRODUCTION}

Collections of emitters coupled to waveguides offer a promising platform for scalable quantum information processing. ${ }^{1-4}$ The applications of such systems range from building long-ranged quantum networks, ${ }^{5,6}$ quantum memory devices,${ }^{7-9}$ and metrology, ${ }^{10}$ to accessing new parameter regimes with exotic light-matter interactions, ${ }^{11,12}$ oftentimes governed by non-Markovian effects.

Non-Markovian dynamics is far richer than the more widely studied Markovian regime. Its different physical origins encompass strong coupling between system and bath, initial system bath correlations, structured reservoirs, and time-delayed coherent feedback, among others. ${ }^{16,17,19-22}$ Non-Markovian collective dynamics due to structured bath and strong coupling has been previously studied in Refs. ${ }^{23-28}$

Another example is the situation where the separations between emitters become comparable to the coherence length of the photons mediating their interaction. Interference effects associated with the phase properties of the electromagnetic (EM) field can then be modified by group dispersion of the photon wavepackets. In such cases, the retarded backaction of the EM field on the atoms leads to a coherent time-delayed feedback on the system dynamics ${ }^{13,14}$ making it non-Markovian. ${ }^{15-18}$ This is the situation that we consider here.

We consider specifically a geometry where two two-state atoms are coupled to an optical waveguide, as depicted in Fig. 1. In such a system where the distance between the atoms can be made quite large, one can consider the situation where the atoms are far enough apart that the retardation effects and finite coherence time scales of the photons mediating their interaction become significant. As a remarkable feature of the dynamics, it has been shown that after the field propagates back and forth between the two atoms it 'learns' that they act as an optical cavity in which it then remains trapped, as in the case of a bound state in continuum (BIC) discussed in Ref. ${ }^{30,31}$ We study here the formation of such a state starting from an initial antisymmetric state of the two atoms.

Further author information:

K.S.: E-mail: kanu@umd.edu

P.M.: E-mail: pierre@optics.arizona.edu

P.S.: E-mail: solano.pablo.a@gmail.com

Quantum Nanophotonic Materials, Devices, and Systems 2019, edited by Cesare Soci, Matthew T. Sheldon, Mario Agio, Proc. of SPIE Vol. 11091, 1109100 - @ 2019 SPIE · CCC code: 0277-786X/19/\$21 · doi: 10.1117/12.2530927 


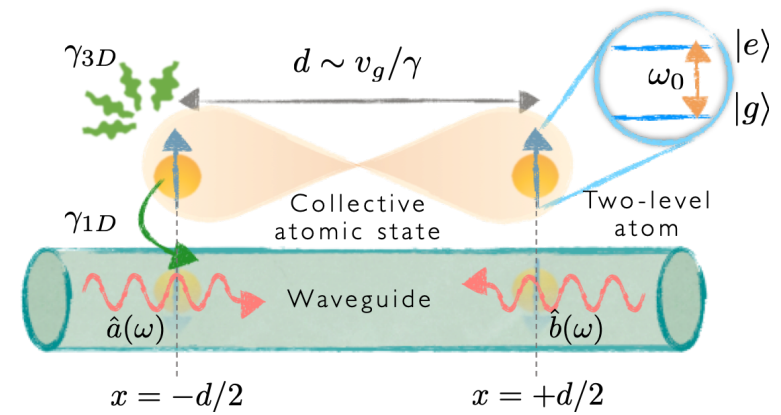

Figure 1. Schematic representation of two atoms coupled to the waveguide. We consider the parameter regime where the interatomic separation is comparable to the coherence length of a spontaneously emitted photon into the waveguide. The atoms are located at positions $x_{1}, 2= \pm d / 2$ along the waveguide such that $d \sim v_{g} / \gamma$, where $v_{g}$ is the group velocity of the field in the waveguide and $\gamma$ is the total spontaneous emission.

\section{MODEL}

To analyze this situation in detail we assume that the two atoms are located at positions $x_{1}=d / 2$ and $x_{2}=-d / 2$ along the length of the waveguide, and share the same radial and azimuthal coordinates for simplicity. In the interaction picture we can write the atoms-field interaction Hamiltonian as

$$
H_{\mathrm{int}}=\hbar \sum_{m=1,2} \int_{0}^{\infty} \mathrm{d} \omega g(\omega)\left[\hat{\sigma}_{+}^{(m)}\left\{\hat{a}(\omega) e^{i \omega x_{m} / v_{g}}+\hat{b}(\omega) e^{-i \omega x_{m} / v_{g}}\right\} e^{-i\left(\omega-\omega_{0}\right) t}+\text { h.c. }\right]
$$

where $\hat{a}(\omega)$ and $\hat{b}(\omega)$ are the annihilation operators for the right- and left-propagating field modes in the waveguide, and $v_{g}$ stands for the group velocity of light in the waveguide.

We assume that the field is initially in the vacuum state and that the atoms share only one quantum of excitation. Specifically, we consider the initial state to be an anti-symmetric state of the two atoms, the so-called subradiant state, with the field in vacuum state, such that $|\Psi(0)\rangle=\frac{1}{\sqrt{2}}(|e, g,\{0\}\rangle-|g, e,\{0\}\rangle)$. The state of the atom + field system at time $t>0$ is then

$$
|\Psi(t)\rangle=\sum_{m=1,2} c_{m}(t) \hat{\sigma}_{+}^{(m)}|g, g,\{0\}\rangle+\int_{0}^{\infty} \mathrm{d} \omega\left[c_{a}(\omega, t) \hat{a}^{\dagger}(\omega)+c_{b}(\omega, t) \hat{b}^{\dagger}(\omega)\right]|g, g,\{0\}\rangle,
$$

where $|g, g,\{0\}\rangle$ refers to the state where both the atoms are in the ground state and the field is in vacuum. Considering that the total Hamiltonian preserves the overall number of excitations in the atom+field system, the above state describes the most general state that the total system can evolve to when starting in a single excitation manifold.

From the Schrödinger equation one can then write the time evolution of the probability amplitudes appearing in $(2)$ as

$$
\begin{aligned}
\dot{c}_{a}(\omega, t) & =-i \sum_{m=1,2} c_{m}(t) g(\omega) e^{-i \omega x_{m} / v_{g}} e^{i\left(\omega-\omega_{0}\right) t}, \\
\dot{c}_{b}(\omega, t) & =-i \sum_{m=1,2} c_{m}(t) g(\omega) e^{i \omega x_{m} / v_{g}} e^{i\left(\omega-\omega_{0}\right) t}, \\
\dot{c}_{m}(t) & =-i \int_{0}^{\infty} \mathrm{d} \omega g(\omega) e^{-i\left(\omega-\omega_{0}\right) t}\left[c_{a}(\omega, t) e^{i \omega x_{m} / v_{g}}+c_{b}(\omega, t) e^{-i \omega x_{m} / v_{g}}\right] .
\end{aligned}
$$

The formal integration of (3) and (4) yields

$c_{a}(\omega, t)=-i \int_{0}^{t} \mathrm{~d} \tau \sum_{m=1,2} g^{*}(\omega) c_{m}(\tau) e^{-i \omega x_{m} / v_{g}} e^{i\left(\omega-\omega_{0}\right) \tau}, c_{b}(\omega, t)=-i \int_{0}^{t} \mathrm{~d} \tau \sum_{m=1,2} g^{*}(\omega) c_{m}(\tau) e^{i \omega x_{m} / v_{g}} e^{i\left(\omega-\omega_{0}\right) \tau}$, 
and substituting these expressions into (5) gives

$$
\begin{aligned}
\dot{c}_{m}(t) & =-\int_{0}^{\infty} \mathrm{d} \omega|g(\omega)|^{2} \int_{0}^{t} \mathrm{~d} \tau \sum_{n=1,2} c_{n}(\tau) e^{-i\left(\omega-\omega_{0}\right)(t-\tau)}\left[e^{i \omega\left(x_{m}-x_{n}\right) / v_{g}}+e^{-i \omega\left(x_{m}-x_{n}\right) / v_{g}}\right], \\
& =-\frac{\gamma}{2} \sum_{n=1,2} c_{n}\left(t-\frac{\left|x_{m}-x_{n}\right|}{v_{g}}\right) e^{i \omega_{0}\left|x_{m}-x_{n}\right| / v_{g}} .
\end{aligned}
$$

Here we have assumed that the density of modes is flat around the atomic resonance such that $g(\omega) \approx g\left(\omega_{0}\right)$ and have introduced the one-dimensional spontaneous decay rate along the direction of the waveguide $\gamma \equiv 4 \pi\left|g\left(\omega_{0}\right)\right|^{2}$. This gives the equations of motion for the excitation amplitudes of the two atoms as

$$
\begin{aligned}
& \dot{c}_{1}(t)=-\frac{\gamma}{2}\left[c_{1}(t)+c_{2}\left(t-d / v_{g}\right) \Theta\left(t-d / v_{g}\right)\right], \\
& \dot{c}_{2}(t)=-\frac{\gamma}{2}\left[c_{2}(t)+c_{1}\left(t-d / v_{g}\right) \Theta\left(t-d / v_{g}\right)\right],
\end{aligned}
$$

where we have assumed that the phase $\phi_{p} \equiv d \omega_{0} / v_{g}=2 n \pi$ and a perfect coupling between the atoms and the waveguide. We note that the above equations are symmetric under the exchange of the two atoms.

\section{INITIAL SUBRADIANT STATE}

For an initial atomic state $\left|\Psi_{\text {atoms }}(0)\right\rangle=\frac{1}{\sqrt{2}}(|e, g\rangle-|g, e\rangle)$ we have $c_{1,2}\left(0^{+}\right)= \pm \frac{1}{\sqrt{2}}$. Let us then consider the Laplace transform of Eqs. (8),

$$
\begin{aligned}
& s \tilde{c}_{1}(s)=\frac{1}{\sqrt{2}}-\frac{\gamma}{2}\left[\tilde{c}_{1}(s)+e^{-d s / v_{g}} \tilde{c}_{2}(s)\right] \\
& s \tilde{c}_{2}(s)=-\frac{1}{\sqrt{2}}-\frac{\gamma}{2}\left[\tilde{c}_{2}(s)+e^{-d s / v_{g}} \tilde{c}_{1}(s)\right] .
\end{aligned}
$$

This yields readily

$$
\begin{aligned}
\tilde{c}_{1}(s)=-\tilde{c}_{2}(s) & =\frac{1}{\sqrt{2}\left[s+\frac{\gamma}{2}-\frac{\gamma}{2} e^{-d s / v_{g}}\right]} \\
& =\frac{1}{\sqrt{2} \gamma\left[\tilde{s}+1 / 2-e^{-\eta \tilde{s}} / 2\right]} .
\end{aligned}
$$

Consider first the limiting case of $d \rightarrow 0$. In this limit, we also note that $\phi_{0} \rightarrow 0$. This allows us to write $\tilde{c}_{1,2}(s) \rightarrow \frac{1}{\sqrt{2}(s)}$, which corresponds to $c_{1,2}(t) \rightarrow \frac{1}{\sqrt{2}}$, illustrating that the two atoms are perfectly subradiant when coincident with each other. We also see that if the atoms are far apart, $d \rightarrow \infty$ one obtains $\tilde{c}_{1,2}(s)= \pm \frac{1}{\sqrt{2}[s+\gamma / 2]}$. In that limit, the atoms therefore decay at the spontaneous emission rate $\gamma$ for both atoms, a signature of the fact the atoms radiate independently of each other as expected.

For an arbitrary interatomic separation we can write the time dependent amplitudes as

$$
\begin{aligned}
c_{1,2}(t) & = \pm \frac{1}{2 \pi i} \int_{-i \infty+\epsilon}^{+i \infty+\epsilon} \frac{\mathrm{d} s}{\sqrt{2}} \frac{e^{s t}}{s+\frac{\gamma}{2}-\frac{\gamma}{2} e^{-d s / v_{g}}}, \\
& = \pm \frac{1}{2 \pi i} \int_{-\infty-i \epsilon}^{+\infty-i \epsilon} \frac{\mathrm{d} \tilde{s}}{\sqrt{2} \gamma} \frac{e^{\tilde{s} \gamma t}}{\tilde{s}+\frac{1}{2}-\frac{1}{2} e^{-\eta \tilde{s}}} .
\end{aligned}
$$

The pole of the denominator is determined by the characteristic equation

$$
\tilde{s}+\frac{1}{2}-\frac{1}{2} e^{-\eta \tilde{s}}=0 \Longrightarrow(\eta \bar{s}) e^{\eta \bar{s}}=\frac{\eta}{2} e^{\eta / 2} \Longrightarrow \tilde{s}_{n}=\frac{1}{2}\left[1-\frac{W_{n}\left(\frac{\eta}{2} e^{\eta / 2}\right)}{\eta / 2}\right],
$$


where we have introduced $\bar{s} \equiv \tilde{s}+1 / 2$. Here $W(z)$ is the Lambert $W$-function, or more precisely a set of functions $W_{n}(z)$ comprising the $n$ branches of the inverse relation of the function $f(z)=z e^{z}$, where $z$ is a complex number. In other words $z=f^{-1}\left(z e^{z}\right) \equiv W\left(z e^{z}\right), W(z)=f^{-1}(z)$, and $W_{n}(x)$ is its $n$-th branch. ${ }^{29}$

We can now write the Laurent series expansion of the denominator of the integrand

$$
\frac{1}{\tilde{s}+\frac{1}{2}-\frac{1}{2} e^{-\eta \tilde{s}}}=\sum_{n \in \mathbb{Z}} \frac{\alpha_{n}}{\tilde{s}-\tilde{s}_{n}},
$$

such that

$$
\alpha_{n}=\lim _{\tilde{s} \rightarrow \tilde{s}_{n}} \frac{\tilde{s}-\tilde{s}_{n}}{\tilde{s}+\frac{1}{2}-\frac{1}{2} e^{-\eta \tilde{s}}}=\frac{1}{1+W_{n}\left(\frac{\eta}{2} e^{\eta / 2}\right)},
$$

where we have used the property of the $W$-function that $W\left(z_{0}\right) e^{W\left(z_{0}\right)}=z_{0}$. It can be seen from the definition of the $W$-function that given $W\left(z e^{z}\right)=z$, if we substitute $y \equiv z e^{z}$ in this definition, one obtains $W(y)=z$. Substituting $W(y)=z$ back into $y \equiv z e^{z}$ yields $y=W(y) e^{W(y)}$. This gives

$$
\frac{1}{\tilde{s}+\frac{1}{2}-\frac{1}{2} e^{-\eta \tilde{s}}}=\sum_{n \in \mathbb{Z}} \frac{1}{\left\{1+W_{n}\left(\frac{\eta}{2} e^{\eta / 2}\right)\right\}\left(\tilde{s}-\tilde{s}_{n}\right)},
$$

so that we can write the inverse Laplace transform as

$$
c_{1,2}(t)= \pm \frac{1}{\sqrt{2}} \sum_{n \in \mathbb{Z}} \alpha_{n} e^{-\gamma_{n} t / 2}
$$

where we have defined

$$
\begin{aligned}
\alpha_{n} & \equiv \frac{1}{1+W_{n}\left(\frac{\eta}{2} e^{\eta / 2}\right)} \\
\gamma_{n} & \equiv \gamma\left[1-\frac{W_{n}\left(\frac{\eta}{2} e^{\eta / 2}\right)}{\eta / 2}\right] .
\end{aligned}
$$

Observing that $\gamma_{0}=0$ and that for $n \neq 0, \operatorname{Re} \gamma_{n}>0$, it follows that as $t \rightarrow \infty$, there is a steady state term corresponding to $n=0$ that is given as

$$
c_{1,2}(\infty) \rightarrow \pm \frac{1}{\sqrt{2}(1+\eta / 2)}
$$

One can also express the dynamics of the field amplitudes from Eqs. (6) as

$$
\begin{aligned}
& c_{a}(\omega, t)=-i \sqrt{\frac{\gamma}{4 \pi}} \int_{0}^{t} \mathrm{~d} \tau \sum_{m=1,2} c_{m}(\tau) e^{-i \omega x_{m} / v_{g}} e^{i\left(\omega-\omega_{0}\right) \tau}, \\
& c_{b}(\omega, t)=-i \sqrt{\frac{\gamma}{4 \pi}} \int_{0}^{t} \mathrm{~d} \tau \sum_{m=1,2} c_{m}(\tau) e^{i \omega x_{m} / v_{g}} e^{i\left(\omega-\omega_{0}\right) \tau} .
\end{aligned}
$$

We note that for an initial antisymmetric state, the fields emitted into the left and right going modes have opposite phases such that

$$
c_{a}(\omega, t)=-c_{b}(\omega, t)=-\sqrt{\frac{\gamma}{2 \pi}} \sum_{n \in \mathbb{Z}} \alpha_{n} \sin \left(\frac{k d}{2}\right) \frac{e^{\left[i\left(\omega-\omega_{0}\right)-\gamma_{n} / 2\right] t}-1}{i\left(\omega-\omega_{0}\right)-\gamma_{n} / 2} .
$$

For $t \rightarrow \infty$ this reduces to

$$
c_{a}(\omega, t)=-c_{b}(\omega, t)=\sqrt{\frac{\gamma}{2 \pi}} \frac{1}{(1+\eta / 2)} \sin \left(\frac{k d}{2}\right) \frac{1}{i\left(\omega-\omega_{0}\right)} .
$$


so that can thus write the asymptotic state of the system for $t \rightarrow \infty$ as

$$
\left|\Psi_{\infty}\right\rangle=\frac{1}{\sqrt{2}(1+\eta / 2)}\left[(|e g\rangle-|g e\rangle) \otimes|\{0\}\rangle+\sqrt{\frac{\gamma}{\pi}} \int \mathrm{d} \omega \sin \left(\frac{k d}{2}\right) \frac{1}{i\left(\omega-\omega_{0}\right)}\left[\hat{a}^{\dagger}(\omega)-\hat{b}^{\dagger}(\omega)\right]|g g\rangle \otimes|\{0\}\rangle\right]
$$

\section{BOUND STATE IN THE CONTINUUM}

The bound state in the continuum (BIC) of two separated atoms trapped along a waveguide is given by ${ }^{30}$

$$
\begin{aligned}
\left|\Psi_{\mathrm{BIC}}\right\rangle=\frac{1}{\sqrt{1+\eta / 2}} & {\left[\frac{1}{\sqrt{2}}\{|e g\rangle+|g e\rangle\} \otimes|\{0\}\rangle\right.} \\
& \left.-i \sqrt{\frac{\gamma}{4 v_{g}}} \int_{-d / 2}^{d / 2} \mathrm{~d} x\left\{e^{i k_{0}(x+d / 2)} \hat{a}_{R}^{\dagger}(x)-e^{-i k_{0}(x+d / 2)} \hat{a}_{L}^{\dagger}(x)\right\}\right]|g g\rangle|\{0\}\rangle,
\end{aligned}
$$

where the creation operators in the position basis can be written in terms of the momentum basis expressions as

$$
\begin{gathered}
\hat{a}_{R}^{\dagger}(x)=\sqrt{\frac{v_{g}}{\gamma}} \int_{0}^{\infty} \mathrm{d} k e^{-i k x} \hat{a}^{\dagger}(\omega) \\
\hat{a}_{L}^{\dagger}(x)=\sqrt{\frac{v_{g}}{\gamma}} \int_{0}^{\infty} \mathrm{d} k e^{i k x} \hat{b}^{\dagger}(\omega)
\end{gathered}
$$

Substituting these forms in the BIC expression (29) gives

$$
\begin{aligned}
\left|\Psi_{\mathrm{BIC}}\right\rangle= & \frac{1}{\sqrt{1+\eta / 2}}\left[\frac{1}{\sqrt{2}}\{|e g\rangle-|g e\rangle\} \otimes|\{0\}\rangle\right. \\
& \left.-\frac{i}{2} \int_{0}^{\infty} \mathrm{d} k \int_{-d / 2}^{d / 2} \mathrm{~d} x\left\{e^{-i\left(k-k_{0}\right) x} e^{i k_{0} d / 2} \hat{a}^{\dagger}(\omega)-e^{i\left(k-k_{0}\right) x} e^{-i k_{0} d / 2} \hat{b}^{\dagger}(\omega)\right\}\right]|g g\rangle|\{0\}\rangle \\
= & \frac{1}{\sqrt{1+\eta / 2}}\left[\frac{1}{\sqrt{2}}\{|e g\rangle+|g e\rangle\} \otimes|\{0\}\rangle\right. \\
& \left.-\frac{i}{2} \int_{0}^{\infty} \mathrm{d} k\left[\frac{e^{i\left(k-k_{0}\right) d / 2}-e^{-i\left(k-k_{0}\right) d / 2}}{i\left(k-k_{0}\right)}\right]\left\{e^{i k_{0} d / 2} \hat{a}^{\dagger}(\omega)-e^{-i k_{0} d / 2} \hat{b}^{\dagger}(\omega)\right\}\right]|g g\rangle|\{0\}\rangle \\
= & \frac{1}{\sqrt{1+\eta / 2}}\left[\frac{1}{\sqrt{2}}\{|e g\rangle+|g e\rangle\} \otimes|\{0\}\rangle-\frac{i}{2} \int_{0}^{\infty} \mathrm{d} \omega \sin \left(\frac{k d}{2}\right) \frac{1}{i\left(\omega-\omega_{0}\right)}\left\{\hat{a}^{\dagger}(\omega)-\hat{b}^{\dagger}(\omega)\right\}\right]|g g\rangle|\{0\}\rangle
\end{aligned}
$$

The overlap between the BIC and the asymptotic state (28) of the atom-field system is therefore

$$
\begin{aligned}
\left|\left\langle\Psi_{\infty} \mid \Psi_{\mathrm{BIC}}\right\rangle\right|^{2} & =\frac{1}{2(1+\eta / 2)^{3}}\left[\frac{1}{2}+\frac{1}{2}+\frac{\gamma}{\pi} \int_{0}^{\infty} \frac{\mathrm{d} \omega}{\left(\omega-\omega_{0}\right)^{2}} \sin ^{2}\left(\frac{\omega d}{2 v_{g}}\right)\right]^{2} \\
\Longrightarrow\left|\left\langle\Psi_{\infty} \mid \Psi_{\mathrm{BIC}}\right\rangle\right|^{2} & =\frac{1}{1+\eta / 2},
\end{aligned}
$$

which is the result recently referred to in Ref. ${ }^{31}$

\section{CONCLUSION}

We have thus calculated the probability of exciting the BIC state starting from an initial subradiant state of two macroscopically separated atoms. We find that for atoms prepared in an appropriate collective internal state, they can behave as effective mirrors that evolve collectively into a Fabry-Pérot-like resonator, with a bound state stored in the cavity formed by the atoms. This behavior depends strongly on both the collective atomic state and on the atomic separation and suggests an intriguing method to store single photons in macroscopic resonators with dynamically controllable mirror characteristics. 


\section{ACKNOWLEDGMENTS}

We are grateful to Elizabeth A. Goldschmidt, Alejandro González-Tudela, Darrick E. Chang, and Giuseppe Calajó for fruitful discussions.

\section{REFERENCES}

[1] K. Nemoto, M. Trupke, S. J. Devitt, A. M. Stephens, B. Scharfenberger, K. Buczak, T. Nöbauer, M. S. Everitt, J. Schmiedmayer, and W. J. Munro, Photonic Architecture for Scalable Quantum Information Processing in Diamond, Phys. Rev. X 4, 031022 (2014).

[2] M. D. Lukin, M. Fleischhauer, R. Coté, L. M. Duan, D. Jaksch, J. I. Cirac, and P. Zoller, Dipole Blockade and Quantum Information Processing in Mesoscopic Atomic Ensembles, Phys. Rev. Lett. 87, 037901 (2001).

[3] N. Y. Yao, L. Jiang, A. V. Gorshkov, P. C. Maurer, G. Giedke, J. I. Cirac, and M. D. Lukin, Scalable architecture for a room temperature solid-state quantum information processor, Nat. Commun. 3, 800 (2012).

[4] K. Hammerer, A. S. Sørensen, and E. S. Polzik, Quantum interface between light and atomic ensembles, Rev. Mod. Phys. 82, 1041 (2010).

[5] H. J. Kimble, The quantum internet, Nature 453, 1023 (2008).

[6] K. Nemoto, M. Trupke, S. J. Devitt, B. Scharfenberger, K. Buczak, J. Schmiedmayer, and W. J. Munro, Photonic Quantum Networks formed from NV ${ }^{-}$centers, Sci. Rep. 6, 26284 (2016).

[7] A. Asenjo-Garcia, M. Moreno-Cardoner, A. Albrecht, H. J. Kimble, and D. E. Chang, Exponential Improvement in Photon Storage Fidelities Using Subradiance and "Selective Radiance" in Atomic Arrays, Phys. Rev. X 7, 031024 (2017).

[8] S. L. Mouradian, T. Schröder, C. B. Poitras, L. Li, J. Goldstein, E. H. Chen, M. Walsh, J. Cardenas, M. L. Markham, D. J. Twitchen, M. Lipson, and D. Englund, Scalable Integration of Long-Lived Quantum Memories into a Photonic Circuit, Phys. Rev. X 5, 031009 (2015).

[9] M. Steger, K. Saeedi, M. L. W. Thewalt, J. J. L. Morton, H. Riemann, N. V. Abrosimov, P. Becker, and H.-J. Pohl, Quantum Information Storage for over 180 s Using Donor Spins in a ${ }^{28} \mathrm{Si}$ "Semiconductor Vacuum", Science 336, 1280 (2012).

[10] J.-W. Zhou, P.-F. Wang, F.-Z. Shi, P. Huang, X. Kong, X.-K. Xu, Q. Zhang, Z.-X. Wang, X. Rong, and J.-F. Du, Quantum information processing and metrology with color centers in diamonds, Front. Phys. 9, 587 (2014).

[11] J. S. Douglas, H. Habibian, C.-L. Hung, A. V. Gorshkov, H. J. Kimble and D. E. Chang, Quantum manybody models with cold atoms coupled to photonic crystals, Nat. Photon. 9, 326 (2015).

[12] D. E. Chang, J. I. Cirac, and H. J. Kimble, Self-Organization of Atoms along a Nanophotonic Waveguide, Phys. Rev. Lett. 110, 113606 (2013).

[13] S. J. Whalen, A. L. Grimsmo, and H. J. Carmichael, Open quantum systems with delayed coherent feedback, Quantum Sci. Technol. 2, 044008 (2017).

[14] A. L. Grimsmo, Time-Delayed Quantum Feedback Control, Phys. Rev. Lett. 115, 060402 (2015).

[15] H.-P. Breuer, and F. Petruccione, Theory of open quantum systems (Oxford University Press, New York, 2002).

[16] H. P. Breuer, E. M. Laine, J. Piilo, and B. Vacchini, Colloquium: Non-Markovian dynamics in open quantum systems, Rev. Mod. Phys. 88, 021002 (2016).

[17] I. de Vega, and D. Alonso, Dynamics of non-Markovian open quantum systems, Rev. Mod. Phys. 89, 015001 (2017).

[18] C. Fleming, and B. L. Hu, Non-Markovian dynamics of open quantum systems: Stochastic equations and their perturbative solutions, Ann. Phys. 327, 1238 (2012).

[19] B. L. Hu, J. P. Paz, and Y. Zhang, Quantum Brownian motion in a general environment: Exact master equation with nonlocal dissipation and colored noise, Phys. Rev. D 45, 2843 (1992).

[20] B. L. Hu, J. P. Paz, and Y. Zhang, Quantum Brownian motion in a general environment. II. Nonlinear coupling and perturbative approach, Phys. Rev. D 47, 1576 (1993). 
[21] R. Vasile, F. Galve, and R. Zambrini, Spectral origin of non-Markovian open-system dynamics: A finite harmonic model without approximations, Phys. Rev. A 89, 022109 (2014).

[22] S. Gröblacher, A. Trubarov, N. Prigge, G. D. Cole, M. Aspelmeyer, and J. Eisert, Observation of nonMarkovian micromechanical Brownian motion, Nat. Commun. 6, 7606 (2015).

[23] I. Thanopulos, V. Karanikolas, N. Iliopoulos, E. Paspalakis, Non-Markovian spontaneous emission dynamics of a quantum emitter near a MoS2 nanodisk, arXiv:1904.09264 (2019).

[24] N. Vats and S. John, Non-Markovian quantum fluctuations and superradiance near a photonic band edge, Phys. Rev. A 58, 4168 (1998).

[25] S. John and T. Quang, Collective Switching and Inversion without Fluctuation of Two-Level Atoms in Confined Photonic Systems, Phys. Rev. Lett. 78, 1888 (1997).

[26] A. González-Tudela and J. I. Cirac, Markovian and non-Markovian dynamics of quantum emitters coupled to two-dimensional structured reservoirs, Phys. Rev. A 96, 043811 (2017).

[27] A. González-Tudela and J. I. Cirac, Quantum Emitters in Two-Dimensional Structured Reservoirs in the Nonperturbative Regime, Phys. Rev. Lett. 119, 143602 (2017).

[28] D. De Bernardis, T. Jaako, and P. Rabl, Cavity quantum electrodynamics in the nonperturbative regime, Phys. Rev. A 97, 043820 (2018).

[29] R. M. Corless, G. H. Gonnet, D. E. G. Hare, D. J. Jeffrey, D. E. Knuth, On the Lambert-W function, Adv. Comput. Math. 5, 329 (1996).

[30] G. Calajó, Y.-L. L. Fang, H. U. Baranger, and F. Ciccarello, Exciting a Bound State in the Continuum through Multiphoton Scattering Plus Delayed Quantum Feedback, Phys. Rev. Lett. 122, 073601 (2019).

[31] Kanupriya Sinha, Pierre Meystre, Elizabeth A. Goldschmidt, Fredrik K. Fatemi, Steven L. Rolston, Pablo Solano, Non-Markovian collective emission from macroscopically separated emitters, arXiv:1907.12017 